\title{
Diastolic strain time as predictor for systolic dysfunction among patients with active breast cancer
}

\author{
Aviram Hochstadt ${ }^{1}$, Joshua Arnold ${ }^{2}$, Roni Rosen ${ }^{1}$, Chen Sherez ${ }^{3}$, Jack Sherez ${ }^{4}$, Liat Mor ${ }^{1}$, \\ Matthew Derakhshesh ${ }^{1}$, Yonatan Moshkovits ${ }^{1}$, Ilan Merdler ${ }^{1}$, Yaron Arbel $^{5}$, Livia \\ Kapusta $^{4}$, Zach Rozenbaum ${ }^{4}$, Yan Topilsky ${ }^{6}$, and Michal Laufer Perl ${ }^{4}$ \\ ${ }^{1}$ Tel Aviv University Sackler Faculty of Medicine \\ ${ }^{2}$ Affiliation not available \\ ${ }^{3}$ Università degli Studi di Napoli Federico II Scuola di Medicina e Chirurgia \\ ${ }^{4}$ Tel Aviv Sourasky Medical Center \\ ${ }^{5}$ Tel Aviv Medical Center \\ ${ }^{6}$ Mayo Clinic
}

June 18, 2020

\begin{abstract}
Background: Although diastolic dysfunction is common among patients treated with cancer therapy, no clear evidence has been shown that it predicts systolic dysfunction. This study evaluated the correlation of longitudinal diastolic strain time (Dst) with the routine echocardiography diastolic parameters and to estimated its role in the early detection of cardiotoxicity among patients with active breast cancer. Methods: Data were collected as part of the Israel Cardio-Oncology Registry (ICOR), a prospective registry enrolling all adult patients referred to the cardio-oncology clinic. All patients with breast cancer, planned for Doxorubicin therapy were included. Echocardiography, including Global longitudinal systolic strain (GLS) and Dst, was assessed at baseline before chemotherapy (T1), during Doxorubicin therapy (T2) and after the completion of Doxorubicin therapy (T3). Cardiotoxicity were determined by GLS relative reduction of [?]15\%. Dst was assessed as the time measured (ms) of the myocardium lengthening during diastole. =diastolic time (ms) measured. Results: Among 69 patients, 67 (97.1\%) were females with a mean age $52 \pm 13$ years. Diastolic strain timeDst measurement was significantly associated with the standard routine diastolic parameters. Significant GLS reduction was observed in 10 (20\%) patients at T3 . Both in a univariate and a multivariate analyses the change in Ds basal time from T1 to T2 emerged to be significantly associated with GLS reduction at T3 $(\mathrm{p}<0.04)$. Conclusions: Among breast cancer patients, Dst time showed high correlation to standard the routine diastolic echocardiography parameters. Relative reductionChange in Ds basal time emerged associated with clinically significant systolic dysfunction as measured by GLS reduction.
\end{abstract}

Conclusions: Among breast cancer patients, Dst showed high correlation to the routine diastolic echocardiography parameters. Change in Ds basal time emerged associated with clinically significant systolic dysfunction as measured by GLS reduction.

Keywords: Diastolic strain, Global longitudinal strain, cardiotoxicity, cardio-oncology

\section{Introduction}


Cardiotoxicity, defined by a left ventricular ejection fraction (LVEF) reduction, is a well-documented side effect of chemotherapeutic treatment regimens, in particular when anthracycline (ANT) agents are used [1]. Echocardiography has been proven to be a useful tool in evaluating the systolic function by LVEF among patients with cancer [2]. However, standard echocardiography alone is not a sensitive tool for detecting subclinical changes that occur before the development of significant irreversible myocardial damage [3]. Therefore, the need for early myocardial injury detection has become critical in managing patients treated with cancer therapy. Recently, the use of 2D speckle-tracking echocardiography (2D-STE), specifically Global longitudinal strain (GLS), as a measurement of subtle changes in left ventricular systolic function, has been shown to be associated with early diagnosis of cardiotoxicity $[4,5]$. Furthermore, both systolic and diastolic function is essential for normal cardiac function [6]. Diastolic dysfunction development is considered common among patients with cancer [7], however, no clear evidence has shown that it can predict future systolic dysfunction and its relationship to early signs of cardiotoxicity is limited. Currently, diastolic function is evaluated by a number of parameters including e', E/e' ratio, E/A ratio, deceleration time (DT) and left atrium volume index (LAVI) [8, 9, 10], however, these routine measurements do not assess the direct relaxation of the LV and have limitations (e.g. angle dependency) [11]. Strain imaging for the evaluation of diastolic function, using global diastolic strain rate, has been shown to have prognostic information regarding cardiovascular morbidity and mortality $[12,13,14]$. However, the use of longitudinal diastolic strain time (Dst), measuring the time of lengthening of the myocardium and specifically the change in Dst during cancer therapies has not been documented yet.

The aim of this pilot study was to evaluate the correlation of Dst with the routine echocardiography diastolic parameters and to estimate the role of Dst change during cancer therapy for the early detection of cardiotoxicity in patients diagnosed with breast cancer.

\section{Methods}

The study population is part of the Israel Cardio-Oncology Registry (ICOR) - a prospective registry enrolling all patients evaluated in the cardio-oncology clinic at Tel Aviv Sourasky Medical Center. All patients signed an informed consent at the first visit in the clinic and are then followed prospectively. The registry was approved by the local ethics committee (Identifier: 0228-16-TLV) and is registered in clinicaltrials.gov (Identifier: NCT02818517).

This cohort evaluated patients diagnosed with breast cancer planned for ANT therapy. All patients underwent at least 2 echocardiographic evaluations, including GLS; at baseline before chemotherapy (T1) and during Doxorubicin (a type of ANT) therapy (T2). A $3^{\text {rd }}$ echocardiography exam (T3), was performed within 3 months after the completion of Doxorubicin therapy.

The exclusion criteria included LVEF $<53 \%$ at T1 and significant GLS relative reduction[?]15\% at T2.

Diastolic strain was measured by the time of lengthening (Dst) (ms) of the myocardium as specified in the next paragraph (Figure 1). The change in Dst was assessed between T1 to T2 and its association for GLS reduction in T3 was evaluated. A clinically significant reduction in GLS was considered as a relative reduction of [?]15\% from T1 to T3, adhered to the standard benchmark set by previous studies [15].

Three standard apical views (4-chamber, 2-chamber, and 3-chamber) were recorded using a General Electric system, model Vivid S70 echocardiogram and were performed by the same vendor, technician and interpreting cardiologist. Routine Left ventricle (LV) echocardiographic parameters included LV diameters, and LVEF [16]. Early trans-mitral flow velocity (E), late atrial contraction (A) velocity, deceleration time (DT) and early diastolic mitral annular velocity (medial and lateral e') were measured in the apical 4-chamber view [17]. The peak E/e' ratio was calculated (septal, lateral and average). Images were acquired using high frame rate ( $>50$ frames/s) [18], and thereafter stored digitally for offline analysis. GLS was measured using 2DSTE software and tracking within an approximately $5 \mathrm{~mm}$ wide region of interest. An end-systolic frame was used to initialize LV boundaries which were then automatically tracked throughout the cardiac cycle. Manual corrections were performed to optimize boundary tracking as needed. Optimization of images for endocardial visualization through adjustment of gain, compress, and time-gain compensation controls was 
done prior to acquisition. Dst was evaluated by measuring manually the time of lengthening (ms) of the myocardium during diastole. According to past studies, showing significant association between diastolic strain rate and the time constant of LV relaxation [11], we evaluated the Dst from the point of aortic valve closure (AVC) throughout the isovolumic relaxation time and early diastolic, until the plateau of the curve (Fig. 1), Dst was assessed in three apical views (2, 3 and 4-chamber), with 6 segments measured per each view, with a total of 18 segments per each exam. Dst change between T1 and T2 was assessed according to the following segments: Average, Anterior, Inferior, Lateral, Septal, Anteroseptal, Posterior, Apex, Mid and Base, as specified in Table 2.

\section{Statistical Analysis}

Assessment of the relationship between the routine echocardiographic diastolic parameters and 2D-STE derived parameters was done using repeated measures mixed linear regression models with e' or E/e' as the dependent variable, strain measurements as fixed independent variables, and patient serial number as the random variable. To assess the predictive ability of Dst on significant GLS reduction, individual logistic regression models were built with significant GLS reduction as the dependent variable and relative change in each Dst segment between T1 and T2 as independent variables. Using the results of the above models the best predictor of significant GLS reduction was used in a multivariate model to assess its ability to independently predict significant GLS reduction. First, a multivariate model was built with covariates including relative GLS reduction between T1 and T2, baseline cardiac risk factors, cardiotoxic chemotherapy used and cardio-protective medication used. The above primary model was then narrowed using a stepwise forward and backwards Akaike information criterion (AIC) based method in order to select the best predictive model which has lowest AIC. To further illustrate the diagnostic predictive power of Dst alone or in combination with the other model covariates receptor-operator (ROC) curves were built and AUC with 95\% CI and Youden indexes were calculated. Comparison between AUC of ROC curves was done using the DeLong \& DeLong method. To detect whether adding Dst data contributed to the multivariate model predictive ability, net classification index (NRI) was calculated for a logistic model with and without the added variable, $95 \%$ confidence interval for NRI (and its positive and negative components) was calculated using a bootstrapping method. Continuous variables are shown as mean $\pm \mathrm{SD}$, while discrete variable as $\mathrm{n}(\%)$. Results were considered significant when $\mathrm{p}<0.05$. As this is a primary proof of concept investigation, all assessments were considered hypothesis generating and were not corrected for multiple comparisons. All calculations were done using R version 3.5.0, R Foundation for Statistical Computing, Vienna, Austria.

\section{Results}

From July 2016 to March 2019, 72 patients were evaluated, of which 3 patients were excluded due to significant GLS reduction at T2, leaving 69 patients for analysis, with a mean age of $52 \pm 13$ years and female predominance $(97 \%)$. While only $2(3 \%)$ patients had ischemic heart disease, cardiovascular risk factors (hypertension, hyperlipidemia and diabetes mellitus) were relatively common ranging from 12 to 30 percent of the patients. Due to comorbidities 21 patients $(30 \%)$ were treated with either angiotensin converting enzyme inhibitor (ACEI), angiotensin II receptor blocker (ARB) or beta blockers (BB) and 11 (16\%) with statins. All baseline characteristics are summarized on Table 1.

Cancer therapy, other than ANT (100\%), included paclitaxel (88\%) and the recombinant humanized monoclonal antibodies against HER2 Trastuzumab (21\%) and Pertuzumab (19\%) (Table 1).

All patients underwent 2D-STE assessment at T1 and T2 and 50 patients underwent 2D-STE assessment at T3 as well. All patients had normal baseline LVEF (mean $60 \pm 1 \%$ ) and normal GLS (mean $-21 \pm 2 \%$ ). Clinically significant reduction of GLS was observed in 10 (20\%) patients at T3, however only $2(3 \%)$ patients showed a significant reduction of LVEF[?]10\% (Table 1) (7).

The mean baseline Dst (ms) of all 69 patients was $217.01+-28.77 \mathrm{~ms}$ and is presented in Supplementary Table S1according to each segment. When comparing the values of baseline Dst between patients with vs. without cardiac disease or cardiac risk factors, we observed longer Dst values among the latter (Supplementary Table S2). When comparing the Dst change between T1 and T2 we observed a longer values of Dst at T2 among the 
group of patients that developed GLS reduction at T3 (Supplementary Table S3), which was not consistent among the group of patients that did not developed GLS reduction (Supplementary Table S4).

When comparing the association between Dst to e' average, significant inverse correlations were noted in average, anteroseptal, apical and middle segments. When comparing the association of Dst and E/e' average, significant positive correlations were seen in average, lateral, posterior and basal segments (Table 2).

Using logistic models to assess the predictive ability of the relative Dst change from $\mathrm{T} 1$ to T2, the only measurement that showed significant prediction capabilities for significant GLS reduction, was the basal segment time (OR 1.09 for every $1 \%$ increase in basal diastolic time, p=0.03, Table 3). Therefore, we continued investigation of this predictor only, in a multivariate fashion.

After construction of a multivariate logistic regression model of significant GLS reduction between T1 and T3, with covariates including baseline cardiac risk factors, cardiotoxic cancer therapy and cardioprotective medication, we ended up with a final multivariate model that included relative change in basal Dst, relative change in GLS, hypertension, hyperlipidemia and Pertuzumab therapy. Of those, the only significant predictors were the relative change in the basal Dst (OR 1.3 per $1 \%$ change, $\mathrm{p}=0.022$ ) and Pertuzumab treatment (OR 159.1, p=0.035). (Table 4)

The predictive ability of basal Dst for significant GLS reduction between T1 and T3 was moderate with a Youden index of 0.38 and an AUC of 0.732 (95\% CI 0.523-0.940). When building a ROC curve for the prediction of the multivariate logistic model, predictive ability was better with a Youden index of 0.8 and an AUC of 0.950 (95\% CI 0.888- 1) ( $\mathrm{p}$ for AUC comparison = 0.05, Fig. 2). The net reclassification index (NRI) for basal Dst added to the multivariate logistic model was 0.48 (95\% CI of 0.10 to 0.0 .86 ) composed of a positive NRI of 0.50 (95\% CI 0.12 to 0.87 ) and a negative NRI of -0.02 (95\% CI - 0.08 to 0.00 ) showing that adding the basal Dst to the multivariate model was overall significantly beneficial to its predictive ability.

\section{Discussion}

Past studies have shown that reduced GLS is associated with LVEF reduction among patients with cancer [19]; however, limited data is available regarding diastolic strain. It has also been shown that diastolic strain rate is associated with cardiovascular morbidity and mortality [12], but information is still lacking in cancer patient populations [20]. In this study, new methods were highlighted by measuring Ds lengthening time to gain direct insight into the relaxation of the ventricles rather than indirect measurements of filling pressures, to help understand the relationship between diastolic strain changes and overall cardiac dysfunction in patients with breast cancer.

Dst was found to be significantly associated with average e' and average E/e', showing that worse diastolic function by the routine measurements is associated with longer Dst. These correlations suggest that Dst can be used as measurements of diastolic function with an advantage of circumvent the inherent limitations of the routine measurements, such as load and angle dependence [21,22, 23]. The association of longer Dst and cardiac damage was also supported by the longer Dst values observed among patients with vs. without cardiac disease or cardiac risk factors and among patients developing significant GLS reduction. The importance of detection of diastolic dysfunction is apparent when considering its association with increased all-cause mortality in heart failure (HF) patients [24]. However, the early detection and usefulness of 2D-STE measurement techniques among cancer patients have not been well-defined and require further study.

With the use of both a univariate and a multivariate analyses, it was found that the change in basal Dst from T1 to T2 appeared to be significantly associated with a clinically significant GLS reduction at T3 $(\mathrm{p}<0.04)$. When building ROC curves the predictive ability of basal Dst was moderate with an AUC of 0.732, while the entire model including basal Dst showed an excellent predictive ability with an AUC of 0.950, highlights that added to the other variables in the regression basal Dst was overall significantly beneficial to its predictive ability.

In line with previous studies [19, 25], we found that treatment with Pertuzumab emerged also as significantly associated with GLS reduction. Since treatment with Pertuzumab is considered to be less cardiotoxic as 
compared to Transuzumab [1], we believe that future studies focusing on Pertuzumab are required.

The usefulness in predicting the development of cardiac dysfunction can affect treatment course and clinical decision-making as it has been shown that reversing cardiotoxicity can be achieved with early detection and implementation of cardio-protective treatment [26]. Currently, many methods in assessing heart function are considered to be inaccurate, leading to misguidance in therapy and possible premature termination [27].2DSTE has emerged as having the highest sensitivity in detecting early LV changes associated with future dysfunction $[28,29,30]$. The measurement of Dst can contribute to the clinician's predictive capabilities and clinical picture of the cancer patients' heart functioning.

To our knowledge, our study is the first to report the evaluation of Dst. Moreover, data evaluating diastolic strain and its association with systolic dysfunction among cancer patients are limited [20].

Our study has several limitations. First, it was a single center study, however, its strength is the prospective nature following a homogenous population and the unity of all echocardiography performed by the same vendor, technician and interpreting cardiologist. Second, the relatively short period of follow up did not allow us to assess development of LVEF reduction, cardiac morbidity and all-cause mortality. Finally, having many comparisons may increase the chances of having a statistically significant result by chance. However, a physiologic mechanism and consistency in the results make this new method of cardiac function estimation very promising as being another tool in a cardiologist arsenal for risk estimation. A larger cohort with a longer echocardiography follow-up is planned and will allow us to determine the impact of Ds change on the development of LV dysfunction and symptomatic heart failure.

Using a novel measurement method of Dst yielded significant findings. A high correlation was found between the routine diastolic parameters and Dst. Moreover, a relative prolongation of Ds basal time acted as a significant independent early predictor for clinically significant systolic dysfunction as measured by GLS reduction. Future studies are needed in order to strengthen the validity of this new measurement and assessing whether this early marker may play a role in introducing cardio-protective regimens in order to prevent LV dysfunction.

\section{Acknowledgement}

Dr Michal Laufer Perl received a research grant from Pfizer pharmaceuticals Israel Ltd to support the ICOR registry during 2019 .

The other authors have no conflict of interest to declare.

\section{References}

1. Zamorano JL. Lancellotti P, Munoz DR, et al; ESC Scientific Document Group. 2016. ESC Position Paper on cancer treatments and cardiovascular toxicity developed under the auspices of the ESC Committee for Practice Guidelines. Eur Heart J 2016:37:2768-280.

2. Jurcut R, Wildiers H, Ganame J, et al. Strain rate imaging defects early cardiac effects of pegylated liposomal Doxorubicin as adjuvant therapy in elderly patients with breast cancer. J Am Soc Echocardiogr 2008:21:1283-9.

3. Santoro C, Arpino G, Esposito R, et al. 2D and 3D strain for detection of subclinical anthracycline cardiotoxicity in breast cancer patients: a balance with feasibility. Eur Heart J - Cardiovasc Imaging 2017:18(8):930-936.

4. Sawaya H, Sebag IA, Plana JC, et al. Assessment of echocardiography and biomarkers for the extended prediction of cardiotoxicity in patients treated with anthracyclines, taxanes, and trastuzumab. Circ Cardiovasc Imaging 2012:5:596-603.

5. Geyer H, Caracciolo G, Abe H, et al. Assessment of myocardial mechanics using speckle tracking echocardiography: fundamentals and clinical applications. J Am Soc Echocardiography 2010;23:351-369. 
6. Lester SJ, Tajik AJ, Nishimura RA, et al. Unlocking the mysteries of diastolic function: deciphering the Rosetta Stone 10 years later.J Am Coll Cardiol 2008:51:679-89.

7. Plana JC, Galderisi M, Barac A, et al. Expert Consensus for Multimodality Imaging Evaluation of Adult Patients during and after Cancer Therapy: A Report from the American Society of Echocardiography and the European Association of Cardiovascular Imaging. J Am Soc Echocardiogr 2014:911-39.

8. Nagueh SF, Middleton KJ, Kopelen HA, et al. Doppler tissue imaging: A noninvasive technique for evaluation of left ventricular relaxation and estimation of filling pressures. J Am Coll Cardiol 1997:30:152733.

9. Ommen SR, Nishimura RA, Appleton CP, et al. Clinical utility of Doppler echocardiography and tissue Doppler imaging in the estimation of left ventricular filling pressures: A comparative simultaneous Dopplercatheterization study. Circulation 2000:102:1788-94.

10. Dokainish H, Zoghbi WA, Lakkis NM, et al. Optimal non-invasive assessment of LV filling pressures: A comparison of tissue Doppler echocardiography and BNP in patients with pulmonary artery catheters. Circulation 2004:109:2432-9.

11. Nagueh SF, Smiseth OA, Appleton CP, et al. Recommendations for the Evaluation of Left Ventricular Diastolic Function by Echocardiography: An Update from the American Society of Echocardiography and the European Association of Cardiovascular Imaging. J Am Soc Echocardiogr 2016:Apr;29(4):277-314.

12. Lassen MCH, Biering-Sorensen SR, Olsen FJ, et al. Ratio of transmitral early filling velocity to early diastolic strain rate predicts long-term risk of cardiovascular morbidity and mortality in the general population. Eur Heart J 2019:Feb7;40(6):518-525.

13. Ersboll M, Andersen MJ, Valeur N, et al. Early diastolic strain rate in relation to systolic and diastolic function and prognosis in acute myocardial infarction: a two-dimensional speckle-tracking study. Eur Heart J Volume 35, Issue 10, 7 March 2014, Pages 648-656.

14. Morris DA, Boldt LH, Eichstadt H, et al. Myocardial systolic and diastolic performance derived by 2dimensinoal speckle tracking echocardiography in heart failure with normal left ventricular ejection fraction. Circ Heart Fail 2012:Sep1;5(5):610-20.

15. Ponikowski P, Voors AA, Anker SD, et al; ESC Scientific Document Group. ESC Scientific Document Group. 2016 ESC Guidelines for the diagnosis and treatment of acute and chronic heart failure: The Task Force for the diagnosis and treatment of acute and chronic heart failure of the European Society of Cardiology (ESC) Developed with the special contribution of the Heart Failure Association (HFA) of the ESC. Eur Heart J Volume 37, Issue 27, 14 July 2016,2129-2200.

16. Lang RM, Bierig M, Devereux RB, et al; American Society of Echocardiography's Nomenclature and Standards Committee; Task Force on Chamber Quantification; American College of Cardiology Echocardiography Committee; American Heart Association; European Association of Echocardiography, European Society of Cardiology. Recommendations for chamber quantification. Eur J Echocardiogr 2006:7:79-10817.

17. Teo SG, Yang H, Chai P, et al. Impact of left ventricular diastolic dysfunction on left atrial volume and function: a volumetric analysis. Eur J Echocardiogr 2010:11:38-43

18. Charbonnel C, Convers-Domart R, Rigaudeau S. Assessment of global longitudinal strain at low-dose anthracycline-based chemotherapy, for the prediction of subsequent cardiotoxicity. Eur Heart J Cardiovasc Imaging 2017:18:392-401.

19. Laufer-Perl M, Arnold JH, Mor L, et al. The association of reduced global longitudinal strain with cancer therapy-related cardiac dysfunction among patients receiving cancer therapy. Clin Res Cardiol 2019:Jun18.

20. Stoodley PW, Richards DA, Boyd A, et al. Altered left ventricular longitudinal diastolic function correlates with reduced systolic function immediately after anthracycline chemotherapy. Eur Heart J Cardiovasc 
Imaging 2013:March;14(3):228-34.

21. Park JH, Marwick TH. Use and Limitations of E/e' to Assess Left Ventricular Filling Pressure by Echocardiography. J Cardiovasc Ultrasound 2011:Dec;19(4):169-173.

22. Wang J, Khoury DS, Thohan V, et al. Global diastolic strain rate for the assessment of left ventricular relaxation and filling pressures. Circulation 2007:Mar 20;115(11):1376-83.

23. Lee JH, Park JH. Strain Analysis of the Right Ventricle Using Two-dimensional Echocardiography. J Cardiovasc Imaging 2018:Sep;26(3):111-124.

24. Redfield MM, Jacobsen SJ, Burnett JC, et al. Burden of systolic and diastolic ventricular dysfunction in the community: appreciating the scope of the heart failure epidemic. JAMA 2003:289:194-202.

25. Laufer-Perl M, Derakhshesh M, Milwidsky A, et al. Usefulness of global longitudinal strain for early identification of subclinical left ventricular dysfunction in patients with active cancer. Am J Cardiol 2018;00:1-6.

26. Ohtani K, Fujino T, Ide T, et al. Recovery from left ventricular dysfunction was associated with the early introduction of heart failure medical treatment in cancer patients with anthracycline-induced cardiotoxicity. Clin Res Cardiol 108:600-611.

27. Tocchetti CG, Cadeddu C, Di Lisi D, et al. From Molecular Mechanisms to Clinical Management of Antineoplastic Drug-Induced Cardiovascular Toxicity: A Translational Overview. Antioxid Redox Signal 2019:30(18):2110-2153.

28. Hare JL, Brown JK, Leano R, et al. Use of myocardial deformation imaging to detect preclinical myocardial dysfunction before conventional measures in patients undergoing breast cancer treatment with trastuzumab. Am Heart J 2009:158:294-301.

29. Ho E, Brown A, Barrett P, et al. Subclinical anthracycline- and trastuzumab-induced cardiotoxicity in the long-term follow-up of asymptomatic breast cancer survivors: a speckle tracking echocardiographic study. Heart 96:701-707,2010.

30. Pizzino F, Vizzari G, Qamar R, et al. Multimodality imaging in cardiooncology. J Oncol 2015:263950.

Figure 1 legend: Measurement of Diastolic strain time (ms).

A figure that shows how Diastolic strain time (ms) was calculated.

Figure 2 legend: ROC curves for change in Basal Diastolic time alone and as part of the multivariate model for prediction of significant global longitudinal strain (GLS) decrease.

A graph showing ROC curves for predicting significant GLS change between the third and first echo using (green) Basal diastolic time (Dst) change alone with an AUC of 0.726, 95\% CI [0.515, 0.937] and (blue) using the entire Multivariate model with an AUC of $0.950,95 \% \mathrm{CI}[0.888,1]$, p value for differences between AUCs: 0.0

AUC of the ROC for Basal Dst change alone showed statistically significant, although modest comparing to the entire model, supporting that other parts of the model show a different aspect of GLS reduction prediction.

Abbreviations : AUC, area under curve; CI, confidence interval; GLS, global longitudinal strain; ROC, receiver operating characteristic; 
Figure 1: Measurement of Diastolic strain time (ms)

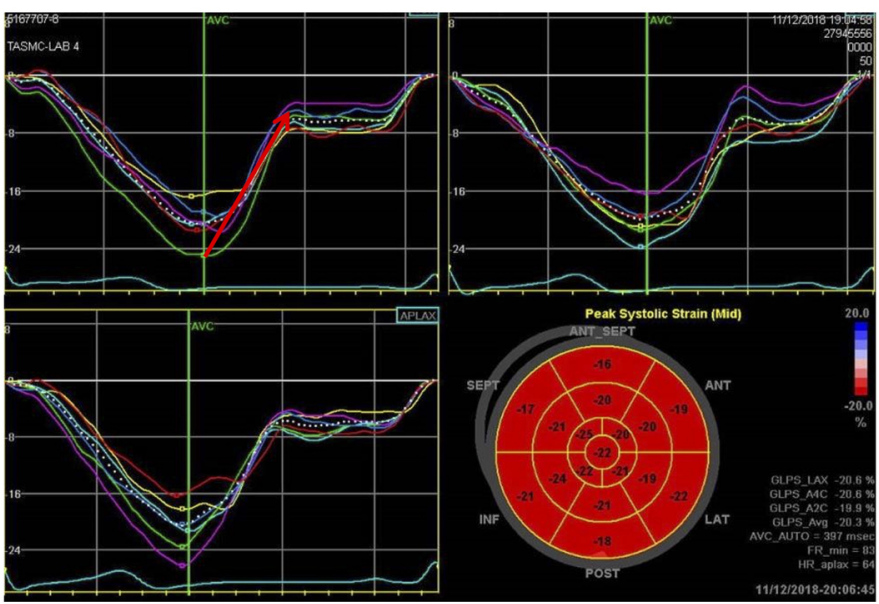

Figure legend: A figure that shows how Diastolic strain time (ms) was calculated. 
Figure 2: ROC curves for change in Basal Diastolic time alone and as part of the multivariate model for prediction of significant global longitudinal strain (GLS) decrease

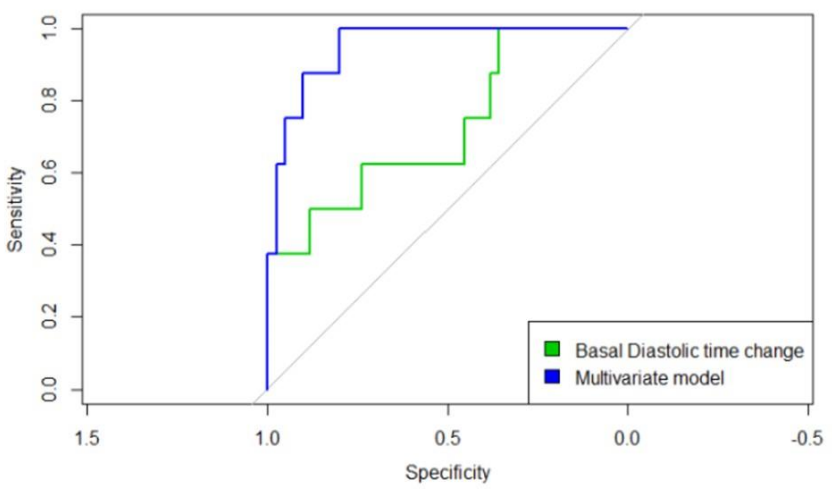

Figure legend: A graph showing ROC curves for predicting significant GLS change between the third and first echo using (green) Basal diastolic time (Dst) change alone with an AUC of 0.726, 95\% CI [0.515, 0.937] and (blue) using the entire Multivariate model with an AUC of $0.950,95 \% \mathrm{CI}[0.888,1], \mathrm{p}$ value for differences between AUCs: 0.0

AUC of the ROC for Basal Dst change alone showed statistically significant, although modest comparing to the entire model, supporting that other parts of the model show a different aspect of GLS reduction prediction. 